\title{
MORFOLOGÍA EXTERNA DE LOS ESTADOS INMADUROS DE Hypothenemus hampei Ferrari (COLEOPTERA: CURCULIONIDAE)
}

\section{EXTERNAL MORPHOLOGY OF THE IMMATURES STAGES OF Hypothenemus hampei Ferrari (COLEOPTERA: CURCULIONIDAE)}

\author{
José David Rubio G. ${ }^{1}$ \\ ${ }^{1}$ I.A. Estudiante Maestría en Entomología, Universidad Nacional de Colombia, Sede Medellín. e-mail: josed.rubio@gmail.com
}

Rev. U.D.C.A Act. E Div. Cient. 12 (2): 157-161, 2009

\section{RESUMEN}

Se presenta la descripción de la morfología externa de los estados inmaduros de la broca del café (huevo, larva y pupa), los cuales, se observaron y se dibujaron bajo estéreo-microscopio de luz. Los resultados de este trabajo servirán para identificar y para realizar estudios de morfología comparada con especies emparentadas y permitirán ampliar el conocimiento de la caracterización morfológica de las formas inmaduras de la broca del café, pues solamente se encuentran descripciones relativamente detalladas para estado adulto, desconociendo la importancia de la conformación, forma y segmentación de los estados inmaduros de una plaga tan limitante en el cultivo del café.

Palabras clave: Scolytinae, broca del café, taxonomía, larva.

\section{SUMMARY}

A description of the external morphology of immature stages of the coffee berry borer (egg, larvae and pupae) was made under light microscope; also drawings were prepared of each stage. The results of this study are considered to be useful for research of comparative morphology with related species and increase the knowledge of the morphological characterization of the immature phases of the coffee berry borer. Up to now only taxonomic determinations of this insect in adult stage are known, ignoring the importance the conformation, form and segmentation of the immature stages of a pest so limiting in coffee cultivation represent.

Key words: Scolytinae, coffee berry borer, taxonomy, larvae.

\section{INTRODUCCIÓN}

La subfamilia Scolytinae, antes Scolitidae, es un grupo relativamente grande dentro de la familia Curculionidae, con un número aproximado de 6000 especies descritas (Wood,1986), dentro de las siguientes tribus y subtribus: Tribu Hylesinini, con las Subtribus: Hylastina, Hylesinina, Tomicina, Phrixosomina, Bothrosternina, Phloeotribina, Phloeosinina, Hypoborina y Polygraphina y Tribu Scolytini, con las Subtribus: Scolytina, Micracina, Cactopinina, Ipina, Dryocoetina, Xyloterina, Xyleborina, Cryphalina (Hypothenemus) y Corthylina (Arnett et al. 2002; Wood, 1982). Las larvas y los adultos de los escolítidos son de hábitos alimenticios fitófagos; varias especies de América se alimentan y se reproducen bajo la corteza o internas en la madera, de una variedad de coníferas, árboles caídos y arbustos leñosos. Muchos atacan árboles muertos recientemente o árboles débiles, pero algunas especies tienen la capacidad de atacar y de matar árboles sanos y vigorosos (Stehr, 1987). Además, 
la adaptabilidad de algunas especies ha servido para que se alimenten y se reproduzcan en cuerpos fructíferos o semillas de una amplia variedad de plantas cultivadas y silvestres (Wood, 1982).

Los escolítidos, los platipódidos, los bréntidos, entre otros, son subfamilias comprendidas en la familia Curculionidae. Las larvas de este grupo de escarabajos son difíciles de distinguir a nivel de subfamilia y se caracterizan por tener forma de C, ser apodas y eucéfalas (Stehr, 1987). La tribu Hylesinini es considerada la más primitiva y las larvas se distinguen por tener de tres a cuatro lóbulos o dientes incisivos en cada mandíbula; en cambio, en Scolytini, se presentan de dos a tres lóbulos o un solo diente grueso. Además, Hylesinini muestra tres o cuatro setas en los lóbulos podicales, mientras que Scolytini siempre posee dos setas en estos lóbulos (Lekander, 1968).

Los insectos que pertenecen al género Hypothenemus son de tamaño pequeño, lo que les facilita el ataque a plantas y semillas. La mayoría de las especies son prolíficas y viven en una gran variedad de hospedantes, por lo que estos insectos son fáciles de transportar y distribuir accidentalmente a otros ambientes, lo que, posiblemente, asegura el éxito de dispersión de este grupo de insectos como especies invasoras de gran importancia económica. Las especies $H$. erectus (Le Conte), H. birmanus (Eichhoff), $H$. hampei (Ferrari), $H$. araccae (Hornung), H. africanus (Hopkins), H. setosus (Eichhoff), H. javanus (Eggers), H. bruneus (Hopkins), $H$. columbi (Hopkins) y H. californicus (Hopkins) llegaron a América procedentes del hemisferio oriental y, aparentemente, $H$. obscurus (Fabricius), H. crudiae (Panzer), H. pulverulentus (Eichhoff), H. puvesens (Hopkins) y H. eruditus (Westwood) han sido exportadas de América a otras partes del mundo (Wood, 1982; Arnett et al. 2002).

La larva de H. hampei es una larva típica de Curculionidae, eucéfala, hipognata y ápoda, adaptada a la vida en el interior de tejidos vegetales, en nuestro caso, granos verdes y maduros, de especies vegetales del género Coffea. Las descripciones realizadas, hasta el momento, solamente se han basado en caracteres taxonómicos del estado adulto desconociendo la importancia de la conformación, la forma y la segmentación de los estados inmaduros, de una plaga tan limitante en el cultivo del café.
Por lo anterior, en este trabajo, se tuvo como objetivo realizar la descripción morfológica del huevo, de la larva y de la pupa de la broca del café, permitiendo así ampliar el conocimiento de la caracterización morfológica de las formas inmaduras, de tan importante insecto.

\section{MATERIALES Y MÉTODOS}

Esta investigación, se llevó a cabo en el laboratorio de Entomología del Centro Nacional de Investigaciones de Café "Pedro Uribe Mejía" CENICAFÉ (Plan Alto, Chinchiná, Caldas) de la Federación Nacional de Cafeteros de Colombia.

Los diferentes estados inmaduros de la broca fueron colectados de frutos de café de un estado fisiológico maduro (más de 190 días después de formación del fruto), en predios de la Estación Central Naranjal, del Centro Nacional de Investigaciones de Café.

Estos frutos fueron disecados con la ayuda de bisturí y de pinzas entomológicas, retirando con cuidado los huevos, las larvas y las pupas para su evaluación, a través de un estéreo-microscopio de luz Marca Zeiss Stemi SV8 y aumento, según el segmento o estado inmaduro evaluado. Un total de seis individuos por estado de desarrollo se fotografiaron, se describieron y se midieron sus diferentes regiones, con el apoyo de un ocular provisto de reglilla micrométrica.

Para la descripción de las larvas de cada espécimen, se desprendió la cabeza y se sumergió en hidróxido de potasio al 5\%, para su aclaración; luego, estas estructuras se fijaron en láminas de vidrio, con bálsamo de Canadá. Las descripciones, se realizaron bajo contrastes generados por el estéreo-microscopio. Además, se determinó el número de segmentos torácicos y abdominales, así como el número de setas presentes en estas regiones.

\section{RESULTADOS Y DISCUSIÓN}

El huevo. Es de color blanco brillante, con el corión transparente y de superficie lisa (Figuras 1.1 y 1.2), su diámetro ecuatorial es de aproximadamente (media \pm EE) $0,32 \pm 0,001 \mathrm{~mm}$, en promedio y su diámetro polar promedio es de 0,64 $\pm 0,001 \mathrm{~mm}$. A medida que se presenta el desarrollo embrionario, el corión se va elongando y opacando; estas observaciones, se 
manifiestan alrededor del tercer día de oviposición. La oviposición, se lleva a cabo una vez la hembra construye la galería dentro del fruto, evitando dejar expuestos los huevos a condiciones climáticas externas y a la acción directa de predadores y parasitoides (Cárdenas $\varepsilon$ Posada, 2001).

La larva. En el primer instar (Figura 1.4), la larva presenta un tamaño longitudinal de 0,63 $\pm 0,014 \mathrm{~mm}$ y un diámetro de 0,26 $\pm 0,005 \mathrm{~mm}$ y la larva de segundo instar (Figura 1.3) muestra un tamaño promedio de 1,39 $\pm 0,014 \mathrm{~mm}$ de largo y un ancho, a nivel de la región abdominal, de 0,56 $\pm 0,002 \mathrm{~mm}$, en promedio. La cabeza es del tipo hipognata; el tórax está compuesto de tres segmentos, siendo el protorácico cerca de 2,5 veces más largo que los segmentos meso y metatorácicos; en el abdomen, se distinguen diez segmentos, el último, generalmente reducido y difícil de diferenciar. El cuerpo de la larva es blando y de textura suave, de color blanco crema, apoda, vermiforme y cubierta por setas erguidas
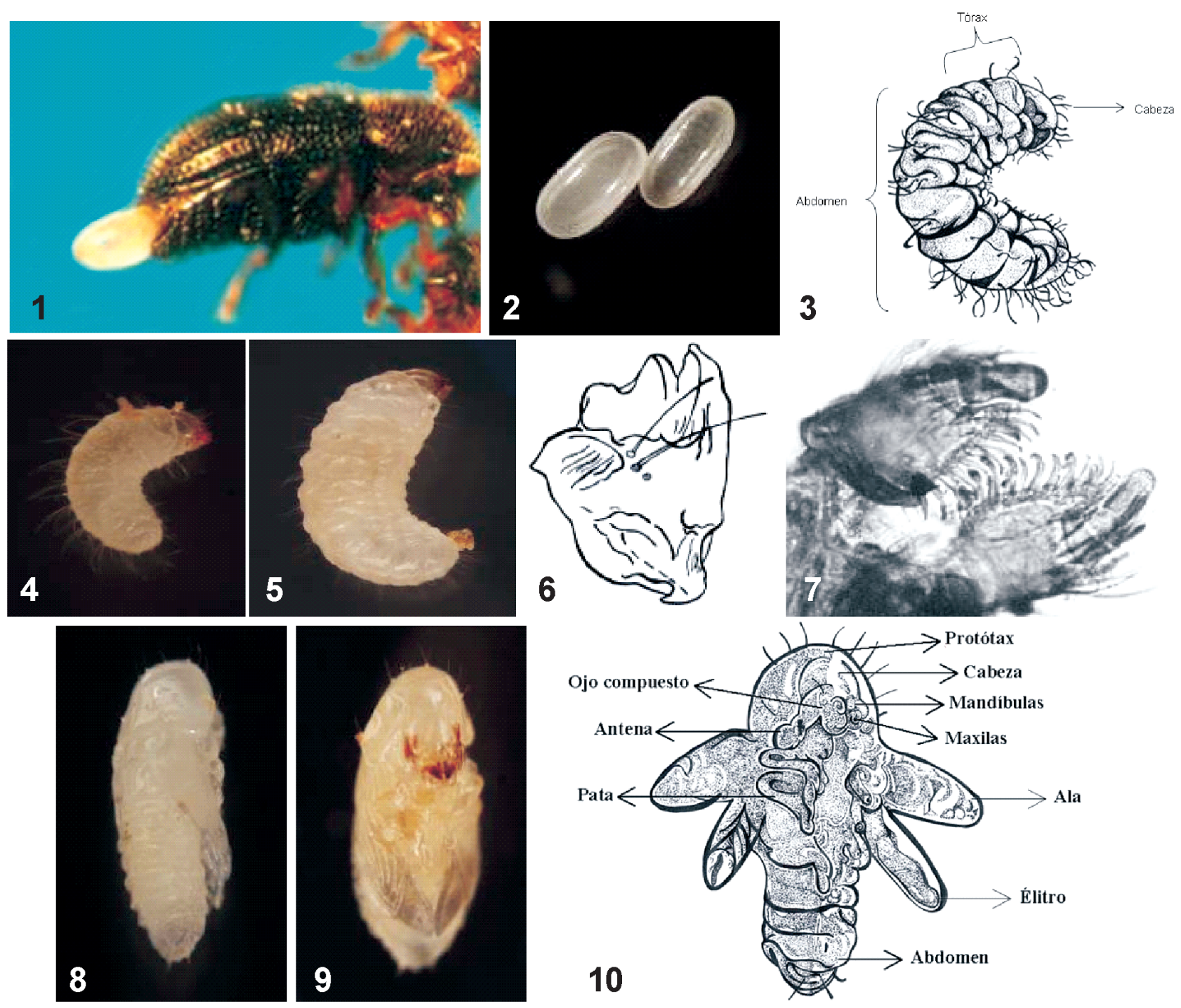

Figura 1. Huevos, estado larval y pupal de Hypothenemus hampei. 1.1) Vista lateral de una hembra en proceso de oviposición; 1.2) vista general de la forma y coloración del huevo, en proceso de desarrollo embrionario; 1.3) vista lateral de la larva de instar dos de $H$. hampei, mostrando los detalles de los pliegues en la región ectodermal; 1.4) vista lateral de la larva del primer instar; 1.5) vista lateral de la larva de segundo instar; 1.6) vista general de la región ventral de la mandíbula izquierda de la larva de segundo instar; 1.7) vista general de las maxilas de larva de segundo instar; 1.8) vista lateral de la pupa; 1.9) vista ventral de la pupa madura, donde se distinguen las mandíbulas en la región anterior de la cabeza y los ojos compuestos laterales a las mandíbulas; 1.10) vista ventral de la pupa de $H$. hampei. 
(Figura 1.5). El sistema traqueal es peripnéustico con un simple par de espiráculos en el protórax y un par en cada uno de los nueve primeros segmentos abdominales. Los espiráculos observados en la larva de $H$. hampei son de tipo anular biforoso, siendo similar a lo observado por Peterson (1960), en larvas de otras especies de la subfamilia Scolytinae.

El aparato bucal de la larva es muy desarrollado y esclerotizado, lo cual, le permite morder y masticar el endospermo duro del café. Las mandíbulas son estructuras esclerotizadas, articuladas dorsolateralmente, situadas en la región anterior de la cabeza y de forma triangular (Figura 1.6); muestra dos regiones: una anterior, la que posee tres dientes incisivos, diseñados para cortar y, la región dorsal, donde existe un molar amplio, que facilita la trituración del material alimenticio; ambas estructuras pareadas, elongadas y algo estrechas en la base, incrementando su tamaño en la región media. Las maxilas están insertadas en la región pleural de la cabeza, debajo de las mandíbulas, y presentan cuatro escleritos, uno proximal o basal, llamado cardo, de forma trapezoidal, grueso y completamente esclerotizado, seguido por el estípete, de color oscuro, esclerotizado, adyacente a la lacinia, que es la zona lobular distal, en cuyos bordes se nota la presencia de gran cantidad de proyecciones dentiformes.

Por último, se localizan los palpíferos con forma cilíndrica, presentes en la zona marginal, que forman el palpo maxilar (Figura 1.7). En esta área, se observa articulado, en posición ventral, el prementum en la región apical, unido al postmentum, por la sutura labial. El labio consta del postmentum en posición basal, unido al cérvix; éste, a su vez, se encuentra dividido en dos: el submentum (proximal) y el mentum (distal). Los palpos labiales, se componen de tres segmentos cada uno, de forma circular, fusionados entre sí y poseen pocas setas. El labro y el clípeo forman un complejo con la epifaringe. Se destaca en las larvas de la broca del café la ausencia de ocelos.

La quetotaxia de la regiones torácicas y abdominales revelan siete pares de setas en las regiones torácicas y en los tergitos abdominales; siete setas a cada lado de la región medio dorsal. Cada uno de los tergitos meso y metatorácicos presenta una seta medio dorsal y tres o cuatro pares de setas cortas. Las setas tergales medio dorsales, se encuentran sobre la línea medio dorsal. La región medio ventral, se observa separada por cinco pares de setas lateroesternales. El noveno segmento muestra tres pares de setas lateroesternales y tergales.

La pupa. Es lisa y muy delicada, inicialmente de color blanco crema (Figuras 1.8 y 1.9) y, a medida que continua el desarrollo, pasan a un color castaño, antes de emerger el adulto. La pupa de $H$. hampei no exhibe ninguna estructura que pueda aportar movilidad, tal como tubérculos o espinas, aunque ellas presentan cierto movimiento abdominal a lo largo del eje longitudinal del cuerpo, al momento de ser perturbadas.

La pupa de la broca es del tipo exarata (Figura 1.10), en la cual, son fácilmente reconocibles los apéndices, como patas, élitros y alas, que se tornan de color oscuro a medida que la pupa madura, lo que facilita el reconocimiento de los ojos compuestos, mediante el uso del microscopio estereoscópico. En esta etapa, se comienza a observar claramente el dimorfismo sexual que, básicamente, se relaciona con el tamaño. La pupa de los machos es más pequeña que la de las hembras. La pupa macho tiene una longitud de 0,88 \pm 0,03mm y un diámetro es de 0,33 \pm $0,007 \mathrm{~mm}$; mientras que el tamaño de las hembras es de $1,18 \pm 0,022 \mathrm{~mm}$ de longitud y su diámetro es de 0,38 $\pm 0,014 \mathrm{~mm}$, en promedio.

La quetotaxia de la pupa es difícil de describir, pero se visualizan ocho setas en el protórax, cuatro anteriores y cuatro posteriores, con un par en la región medio dorsal del meso y metatórax. En la región abdominal no se observaron setas.

Este estudio es el primero sobre la caracterización morfológica completa de los estados inmaduros de la broca del café. El conocimiento de la estructura coriónica y de la morfología larvaria permite establecer caracteres de diagnóstico a nivel de familia, de género y de especie (Crowson, 1981), vital para un diagnóstico acertado de la presencia o no de la broca del café.

AGRADECIMIENTOS: Agradezco a John Albeiro Quiroz Gamboa por los conocimientos y aportes a este manuscrito y a Cenicafé, por la financiación del estudio. Conflicto de intereses: Hago constar que el manuscrito fue preparado y revisado por el autor, quien declara que no existe ningún conflicto de intereses que ponga en riesgo la validez de los resultados presentados. 


\section{BIBLIOGRAFÍA}

1. ARNETT JR., R.H.; THOMAS, M.C.; SKELLEY, P.E.; FRANK, J.H. 2002. American beetles, Vol. 2. Boca Raton (Estados Unidos). CRC Press. 861p.

2. CÁRDENAS M., R.; POSADA F., F.J. 2001. Los insectos y otros habitantes de cafetales y platanales. Comité Departamental de Cafeteros del QuindíoCenicafé. 250p.

3. CROWSON, R.A. 1981. The biology of the Coleoptera. Londres (Inglaterra), Academic Press, 802p.

4. LEKANDER, B. 1968. The number of larval instars in some bark beetle species. Entomol. Tidskr. (Suecia). 89:25-34.

5. PETERSON, A. 1960. Larvae of insects. An Introduction to Nearctic Species Part II. Coleoptera, Diptera, Neuroptera, Siphonaptera, Mecoptera,
Trichoptera. Ohio University. Columbus (Ohio). Ed. Edwards Brothers, Inc. Ann Arbor, Michigan. 416p.

6. STEHR, F.W. 1987. Immature insects. Dubuque (Estados Unidos), Kendall/Hunt Publishing Company, Vol. 2. 794p.

7. WOOD, S.L. 1982. The bark and ambrosia beetles of North and Central America (Coleoptera - Scolytidae), a taxonomic monograph. Great Basin Nat (Utha - Estados Unidos). Mem. 6:1359.

8. WOOD, S.L. 1986. A reclassification of the genera of Scolytidae (Coleoptera). Great Basin Nat. Mem. 10:1-126.

Recibido: Julio 6 de 2009

Aceptado: Octubre 16 de 2009 